\title{
Traduire
}

Une eutre perspective sur $r$ tatadciction

Revue française de la traduction

$221 \mid 2009$

Voies de l'interprétation

\section{Les interprètes sont-ils des tricheurs ?}

\section{Benoît Kremer}

\section{(2) OpenEdition}

Journals

Édition électronique

URL : http://journals.openedition.org/traduire/340

DOI : 10.4000/traduire.340

ISSN : 2272-9992

\section{Éditeur}

Société française des traducteurs

\section{Édition imprimée}

Date de publication : 15 décembre 2009

Pagination : 13-18

ISSN : 0395-773X

\section{Référence électronique}

Benoît Kremer, «Les interprètes sont-ils des tricheurs ? », Traduire [En ligne], 221 | 2009, mis en ligne le 12 novembre 2013, consulté le 25 novembre 2020. URL : http://journals.openedition.org/traduire/340 ; DOI : https://doi.org/10.4000/traduire.340 


\section{Les interprètes sont-ils des tricheurs?}

\section{Benoît Kremer}

De toutes les professions intellectuelles, rares sont celles qui suscitent autant d'incompréhensions et de malentendus que l'interprétation. D'un côté, le grand public ressent une grande admiration pour les personnes, à demi dans l'ombre entre deux chefs d'État, qui leur chuchotent à l'oreille les propos de leurs interlocuteurs. II n'est pas rare que les jeunes rêvent d'exercer une profession auréolée d'un tel prestige, bien qu'ils soient souvent en peine d'expliquer les véritables compétences requises et ignorent comment s'organise le quotidien des interprètes. De l'autre côté, on entend parfois évoquer à mots couverts des critiques que l'on pourrait résumer d'une phrase lapidaire : les interprètes sont des tricheurs ! Qu'il soit ici permis à un représentant de cette profession de "crever l'abcès " en examinant sur le fond les reproches faits aux interprètes (mais souvent indirectement, et rarement formulés en ces termes).

Pour retracer de la façon la plus aisée les divers types de critique adressés aux interprètes, prenons l'exemple (fictif) d'une interprète ${ }^{(1)}$ et suivons-la tout au long des contacts avec son client.

Tout commence avec l'appel téléphonique d'un client potentiel, désireux d'organiser une réunion internationale et plus ou moins obligé(2) de veiller à la bonne communication entre les participants. L'interprète se fait expliquer les besoins (durée et lieu de la réunion, langues parlées par les orateurs, langues vers lesquelles l'interprétation est souhaitée : dans notre exemple, russe et français). TRICHERIE !! s'exclame son interlocuteur : vous prétendez être

(1) L'auteur de ce texte est un homme, mais à ce titre, se retrouve en minorité dans cette profession, exercée à une écrasante majorité par des femmes. On comprendra donc qu'il choisisse une collègue féminine dans son exemple.

(2) II est hélas rare que les organisateurs de conférences souhaitent recruter des interprètes de leur plein gré et avec enthousiasme ; dans leur esprit, l'utilisation d'une langue unique (suivez mon regard) permettrait de faire des économies, même si elle n'est la langue maternelle d'aucun orateur et bien évidemment d'aucun auditeur dans la salle... Mais avec un peu de chance, les enjeux sont trop élevés ou les participants viennent d'horizons trop différents pour que l'on puisse se permettre de risquer de faire capoter la rencontre pour de "simples questions linguistiques ". 
interprète, libre à la date voulue, mais tout à coup vous me dites que vous ne pouvez pas couvrir seule toutes les langues de la réunion! Certes, répond l'interprète, mais d'une part, je travaille exclusivement du russe vers le français, et pas du français vers le russe, donc il faut aussi assurer la communication dans l'autre sens ; et d'autre part, aucun interprète professionnel digne de ce nom n'acceptera jamais de travailler seul en simultanée au-delà d'une demi-heure. TRICHERIE ! lui réplique-t-on, on m'a demandé de recruter un interprète, et voilà que je me retrouve avec toute une équipe de quatre personnes... Cela va me revenir plus cher et je ne suis pas sûr que cela serve à grand-chose... L'interprète s'échine alors à faire comprendre que la question des coûts ne doit pas se voir à travers l'addition des frais engagés pour organiser une réunion, mais à travers la qualité de la communication, la satisfaction des participants et le sentiment général qu'ils n'ont pas perdu leur temps.

Dans un contexte d'économies généralisées, le strict calcul des coûts engagés doit toujours être mis en regard de l'utilité réelle des activités qu'ils rémunèrent. Ainsi, même en période de crise, il est rare de pinailler sur la qualité de l'orateur invité, que l'on fait venir à grands frais de l'autre bout du monde, sur le confort des salles de réunions, dont on n'imagine pas qu'elles se composent de chaises en bois entourées de quatre murs de parpaings coiffés de tôle ondulée, sur l'efficacité du système d'amplification acoustique, qui doit répondre aux normes technologiques les plus modernes, ni même sur l'offre de boissons à la pause café, voire sur la décoration florale. Face à ces coûts, ceux d'une équipe d'interprètes représentent souvent une faible fraction, mais ce sont eux que l'on met en exergue et dont l'organisateur pense aisément qu'ils sont "superflus " ou " excessifs ". II ferait cependant bien mieux de penser à ce que lui coûterait l'absence de participation de ses invités russes, leur insatisfaction ou, pire encore, l'incompréhension totale entre les participants !

Supposons donc que l'organisateur vienne à résipiscence. Parmi les conditions que l'interprète-conseil(3) va réclamer figurent les honoraires et les conditions de voyage. TRICHERIE ! s'exclame à nouveau l'organisateur. Seules les divas d'opéra du passé se permettaient d'exiger tout le confort pour leurs voyages et leur hébergement! Je ne recrute pas des interprètes pour leur offrir des vacances! À ce stade, force est de lui faire comprendre que l'interprétation est une profession intellectuelle exigeant une concentration énorme, impossible à assurer lorsque l'on a passé la nuit dans un train bondé, que l'on n'a pas fermé l'œil parce que la chambre d'hôtel était bruyante ou que l'on a dû se lever dès potron-minet parce que l'hôtel était à l'autre bout de la ville.

(3) On nomme « interprète-conseil " l'interprète qui ne se contente pas d'accepter ou de refuser une offre de travail le concernant directement, mais qui oriente et guide également le client potentiel à travers tout le labyrinthe des questions d'organisation relatives à l'interprétation, en composant l'ensemble de l'équipe et en trouvant les collègues correspondants. Dans les grandes lignes, tout interprète "lambda " peut donner à ses contacts les conseils requis, mais certains interprètes-conseils ont accumulé une grande expérience professionnelle en matière d'orientations à donner en vue d'une organisation professionnelle de la communication dans une réunion internationale. 
La réaction du client risque aussi d'être similaire lorsqu'il lui est expliqué quels sont les besoins techniques des interprètes (cabines répondant aux normes ISO, équipement technique approprié, présence d'un technicien expérimenté). Mais il est relativement plus facile de faire comprendre ces besoins, d'autant que pour une opération non plus, on ne donne pas un couteau de cuisine à un chirurgien, fût-il le meilleur.

Mais les conditions concrètes ne sont pas les seules sur lesquelles l'interprète-conseil doit se battre : nous en arrivons maintenant à des reproches bien plus profonds et qui appelleront une discussion plus sérieuse. L'interprète-conseil aborde en effet la question de la préparation : l'organisateur peut-il lui remettre de la documentation susceptible de l'aider (procès-verbaux antérieurs, glossaires internes, description plus détaillée des enjeux de la réunion, liste des participants, éventuellement texte de leurs interventions) ? TRICHERIE ! Je croyais que pour ce prix, je pouvais dormir sur mes deux oreilles, et voilà qu'il faut encore que je fournisse des documents confidentiels internes, que je fasse, moi, des recherches dans mes archives, et que je donne aux interprètes les moyens concrets de répéter les mots dont ils auront besoin en réunion - à ce compte-là, autant faire le travail moi-même!

Ces trois objections méritent un examen plus poussé. En premier lieu, les interprètes sont tenus au secret professionnel total et absolu. Dès lors, tout ce que l'organisateur leur remet reste strictement confidentiel, ce qui contribue à asseoir la confiance indispensable envers cet organisateur-ci, mais aussi envers tous les organisateurs possibles à l'avenir, qui sauront désormais qu'aucun secret ne filtrera hors de la réunion à cause des interprètes.

De plus, ces documents ne servent qu'à faciliter l'intégration de l'interprète dans la réunion. On peut dire en effet que, pour la durée de l'engagement, l'interprète devient partie intégrante de l'entreprise, du projet, de l'organisation ou de la rencontre ; pour pouvoir s'adapter en si peu de temps, il fournit bien évidemment des efforts personnels, mais ceux-ci seront encore plus efficaces si l'organisateur lui " ouvre la porte " et l'aide, de son côté aussi, à se familiariser avec le jargon maison, les procédures internes, les enjeux cachés.

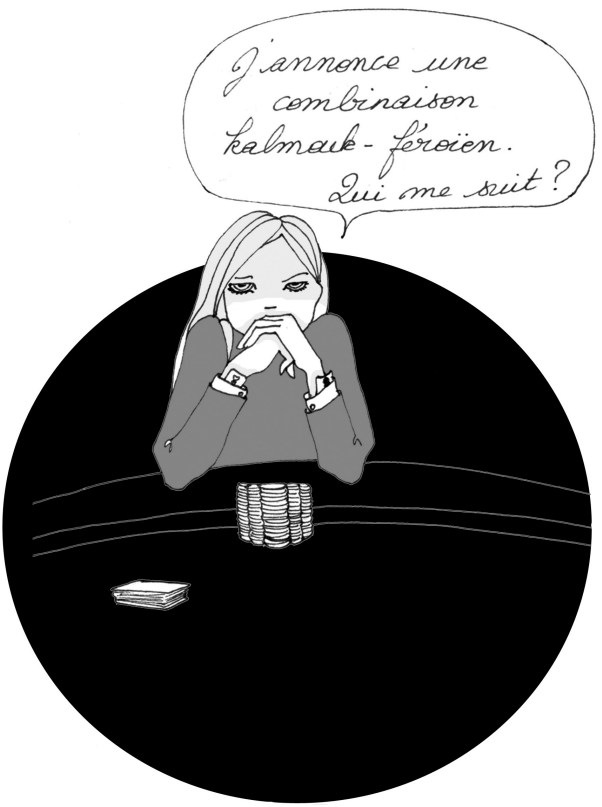

Illustration : Marlène Junius, http://alotoftralala.over-blog.com 
Enfin, il est vrai que les participants à une réunion manient toujours un vocabulaire technique spécialisé, et que les auditeurs de l'interprétation sont en droit d'entendre ce vocabulaire utilisé à bon escient par les interprètes dûment préparés. Mais le plus grand malentendu, dans la situation que nous décrivons, tient à la croyance erronée que l'interprétation consiste à répéter aussi rapidement que possible les mots prononcés par l'orateur. Or, L'INTERPRÉTATION N'EST PAS UNE OPÉRATION SUR LES MOTS, MAIS SUR CE QUI EST DIT À TRAVERS EUX ! DèS lors, les mots sont un moyen, mais pas une fin en soi. Le discours de l'orateur est en effet constitué non seulement de termes techniques, mais aussi de références culturelles, de sousentendus, d'intentions, d'expressions idiomatiques, de tournures personnelles, le tout noyé dans des formulations purement grammaticales. Tout cela exige, de manière instantanée, une analyse sémantique poussée ; ce n'est qu'au terme de celle-ci que l'interprète pourra choisir, dans la langue d'aboutissement, l'habillage qui conviendra pour faire passer le message dans cette autre langue, qui répond à d'autres exigences grammaticales, mais aussi culturelles et de pensée. Si l'on prend conscience de ce que cela exige de connaissances préalables, de concentration, d'esprit d'analyse et de décision, ainsi que d'aisance dans l'expression orale, le tout sous l'effet du stress dû à l'intensité de la pensée de l'orateur, mais aussi à la rapidité des opérations mentales en jeu, on peut comprendre le désir de l'interprète de mettre tous les atouts de son côté pour ne pas compromettre la réunion par une formulation imprécise ou inexacte.

Car il faut aussi voir que certains organisateurs s'imaginent (TRICHERIE ! là encore) que l'interprète ne parvient à tenir le rythme imposé par les orateurs que parce qu'ils se contentent de faire un résumé de leurs propos, qu'ils expriment un contenu sommaire et qu'ils restent en surface de l'intervention dès lors qu'une difficulté se présente. Or, tel n'est évidemment pas le cas - mais le malentendu sur le rôle de l'interprète est là, malgré tout. Je me rappelle avoir entendu deux auditeurs s'étonner, à l'issue d'une réunion, que les interprètes aient " même " su faire passer l'humour et l'ironie du président. Eh bien oui ! Les interprètes ne se contentent pas de transmettre le contenu technique d'un discours, mais aussi l'esprit dans lequel il est prononcé ; évidemment, tout l'éventail de leurs compétences ne ressort que lorsque les discours prononcés s'y prêtent (l'interprète, après tout, respecte fidèlement et pleinement le contenu de celui ou celle qu'il/elle traduit, mais ne peut y mettre plus que ce qui s'y trouve au départ...).

Il faut concéder qu'il n'est pas aisé de prendre conscience en profondeur des processus intellectuels décrits plus haut : pour qui comprend le russe, écouter un orateur s'exprimer dans cette langue est une opération simple et immédiate, mais qui ne prend pas nécessairement la mesure des difficultés propres à l'orateur (a-t-il un accent particulier ? forme-t-il des phrases complètes ? utilise-t-il des expressions rares ou des termes techniques complexes ? son débit est-il excessivement soutenu ? [dans certains cas, celui qui se contente d'écouter peut trouver très plaisant un discours rapide, enlevé, truffé d'anecdotes et de blagues, alors que cela constitue autant de difficultés et d'obstacles pour l'interprète]). De même, pour qui comprend le français, 
suivre l'interprétation française de ce discours ne permet pas de juger de la complexité du processus impliqué (l'interprète a-t-elle dû reformuler toutes les phrases ? faire constamment appel à sa mémoire à court terme ? réorganiser les éléments du discours ? surmonter des difficultés d'ordre linguistique ou technique ?) ; l'auditeur se contente d'apprécier l'aisance de l'interprète, sa voix, la précision de ses formulations, son respect des notions techniques et l'efficacité de sa communication. A fortiori, il ne suffit pas de connaître à la fois le russe et le français pour juger de la qualité de l'interprétation : pour cela, il faudrait écouter d'une oreille l'original et de l'autre l'interprétation, et refaire en pensée le cheminement mental qui aboutit de l'un à l'autre - le tout, en quelques fractions de seconde. Bien entendu, seules des personnes dûment formées (interprètes ou enseignants en interprétation) sont habituées à ce genre d'exercice. Mais pour un client, il ne suffit évidemment pas d'écouter l'orateur, puis de porter les écouteurs pendant cinq minutes afin de voir si l'interprète " suit ", pour juger de la qualité intrinsèque de son travail, ni même pour prendre conscience de ce que ce travail représente. Je concède cependant que c'est le résultat final qui compte, et je serais le premier à avouer qu'il y a TRICHERIE ! et même tromperie sur la marchandise si le " produit fini " livré par l'interprète est une bouillie inaudible. Mais il me faut aussi insister sur le fait qu'un message impeccable, d'un contenu parfait, formulé avec aisance et précision et exprimé sur un ton agréable et communicatif n'est pas le produit d'une répétition de mots et de termes irréfléchie, mais d'une formation poussée et d'une préparation soutenue en amont, ainsi que d'un processus intellectuel complexe et exigeant réalisé dans l'instant.

On touche ainsi du doigt une autre critique, souvent implicite et inavouée (et peut-être presque inavouable !). II y aurait TRICHERIE de la part des interprètes précisément parce que l'on n'a pas l'impression que leur travail soit compliqué : ils voyagent dans le confort, s'installent en cabine, ouvrent le micro, répètent deux ou trois phrases, et le tour est joué - il ne reste plus qu'à rentrer chez soi en passant, bien sûr, par la case " paiement ". Or, l'effort est soutenu, je crois l'avoir décrit plus haut, mais, reconnaissons-le, il est assez peu visible. Cela tient d'une part à ce que l'interprète de qualité fournit une prestation professionnelle qui élimine les hésitations et assure à l'auditeur une écoute agréable et un contenu irréprochable. D'autre part, cela s'explique aussi par le fait que la prestation de l'interprète est fluide et impalpable parce qu'elle ne s'inscrit pas dans la durée, mais dans le moment immédiat : l'interprétation est un produit qui se consomme "à chaud ", dans l'instant de la discussion et dans une situation de communication précise. C'est l'élément qui contribue à la bonne communication, aux échanges entre les participants, à l'instauration du dialogue, mais comme le sel, il se dissout dans le plat et, au moment de la dégustation, n'est plus perçu comme un ingrédient à part, mais comme un élément indissociable du tout. En d'autres termes, c'est précisément lorsque l'interprète n'a pas fait sentir sa présence qu'il s'est avéré le plus efficace !! Et dans ces circonstances, il est particulièrement difficile de dissocier les unes des autres les diverses composantes qui ont contribué à ce succès, parce qu'elles ont été "digérées " au fur et à mesure de la discussion, qui a eu lieu oralement et dont, par conséquent, aucune trace ne demeure à des fins de véri- 
fication ${ }^{(4)}$. Pour résumer, on pourrait citer le proverbe anglais the proof of the pudding is in the eating : c'est le bon déroulement de la réunion (sur le plan de la communication proprement dite, évidemment, et non sur celui des résultats concrets, qui, eux, ne dépendent pas des interprètes !) qui atteste (rétrospectivement) de la qualité de l'interprétation.

Ce n'est donc qu'à la fin de notre réunion prise en exemple que le client pourra s'adresser aux interprètes pour reconnaître qu'il n'y avait pas eu TRICHERIE ! mais que leur intervention avait réellement contribué au succès et à l'entente entre les participants russes et français. Si chaque maillon de la chaîne joue pleinement son rôle, l'interprète-conseil en dispensant des conseils avisés, en guidant le client et en lui recommandant les meilleures solutions, les interprètes individuels en se préparant au mieux et en mettant en œuvre toutes leurs compétences professionnelles, le client lui-même en acceptant d'intégrer les interprètes dans l'organisation de sa réunion et en leur donnant les moyens d'y apporter leur contribution efficace, alors l'utilité de la profession d'interprète sera évidente pour tous.

Par-delà tous les reproches évoqués, il en est un qui ne l'est jamais, alors qu'il pourrait éventuellement être le seul justifié... En effet, de par les contraintes propres au métier, les interprètes répètent à longueur de temps les propos des autres. Mais au fond d'eux-mêmes, ils ressentent souvent le besoin de faire entendre leur propre voix, d'exprimer leur créativité et leurs idées sans passer par celles d'autrui, en d'autres termes de se transformer en orateurs! Se glissant dans la peau des personnes qu'ils traduisent, les interprètes ressemblent à des acteurs qui endossent chaque soir un rôle différent - mais se contentent évidemment de le jouer, sans le vivre réellement. Qui sait, donc, si à ce stade, on ne pourrait pas les accuser à juste titre de faire semblant, c'est-à-dire de tricher?

b.kremer@aiic.net

Benoit Kremer est traducteur indépendant (1981), membre de la SFT, interprète de conférence (1983), enseignant à l'ETI (École de Traduction et d'Interprétation, Université de Genève) (introduction à l'interprétation, consécutive d'anglais en français, simultanée d'allemand en français) et à l'Université de Grenoble (traduction juridique d'allemand en français), ancien président du Conseil de l'ETI (20062007), Président de l'AllC (Association Internationale des Interprètes de Conférence, www.aiic.net) depuis 2006, réélu en 2009.

(4) Bien entendu, cela représente une des différences majeures avec la traduction, dont la trace écrite reste toujours disponible pour un " contrôle " ultérieur à tête reposée, mais aussi avec la contrainte supplémentaire, pour le traducteur, que son travail doit pouvoir résister à des contextes de lecture différents, à des publics différents, voire au passage des années. Autre source, d'ailleurs, d'un certain sentiment de TRICHERIE, l'opération de traduction écrite paraissant ainsi infiniment plus compliquée, mais aussi plus noble, que l'opération de traduction orale, alors que cette dernière reste auréolée d'un plus grand prestige... 